\title{
4-D Motion Field Estimation by Combined Multiple Heart Phase Registration (CMHPR) for Cardiac C-arm Data
}

\author{
Kerstin Müller, Christopher Rohkohl, Günter Lauritsch, Chris Schwemmer, \\ Hein Heidbüchel, Stijn De Buck, Dieter Nuyens, Yiannis Kyriakou, Christoph Köhler, and Joachim Hornegger
}

\begin{abstract}
In interventional cardiology three-dimensional anatomical and functional information of the cardiac chambers would have an important impact on diagnosis and therapy. With the technology of $\mathrm{C}$-arm CT it is possible to reconstruct intraprocedural 3-D images from angiographic projection data. In order to generate accurate and artifact-free reconstructions from dynamic cardiac projections, the motion needs to be taken into account. We present the novel Combined Multiple Heart Phase Registration (CMHPR) method. CMHPR is an iterative motion estimation and compensation algorithm that uses projection data acquired during a single $\mathrm{C}$-arm sweep. Filtered-backprojection (FBP) volumes from electrocardiogram (ECG)-gated data are reconstructed for different motion states of the heart. According to an unknown 4-D motion vector field the ECG-gated FBP images are deformed and accumulated to a sum volume for representing the status of a particular heart phase. In an iterative optimization procedure the 4-D motion vector field is computed by registering the sum volume to a reference volume of the same heart phase. The negative normalized cross correlation (NCC) of both volumes is used as a cost function. In this paper, the reference image is generated using the prior image constrained compressed sensing (PICCS) algorithm combined with the improved total variation (iTV). First preliminary experiments on clinical porcine data sets show promising results. CMHPR reduces streak artifacts and simultaneously preserves sharp edges without producing the artificial comic-like appearance of the PICCS + iTV reference volume.
\end{abstract}

\section{INTRODUCTION}

\section{A. Purpose of This Work}

In the daily clinical routine, characteristic parameters quantifying the ventricular heart wall motion are based on 2D angiographic projections acquired with a C-arm system [1]. These images can be used for visual guidance during interventions and to analyze clinically significant parameters, e.g. ejection fraction and wall motion of the heart chambers. Due to the nature of the projection, the images cannot exhibit depth information. Therefore, a 3-D reconstruction is desirable to measure the parameters directly in the three-dimensional space. $\mathrm{C}$-arm systems have a slow period of rotation (a few

K. Müller, C. Schwemmer and J. Hornegger are with the Pattern Recognition Lab, Department of Computer Science and the Erlangen Graduate School in Advanced Optical Technologies (SAOT), Friedrich-Alexander-Universität Erlangen-Nürnberg, Erlangen, Germany. Email:kerstin.mueller@cs.fau.de. C. Rohkohl, G. Lauritsch, Y. Kyriakou and C. Köhler are with the Siemens AG, Healthcare Sector, Forchheim, Germany. H. Heidbüchel, S. De Buck and D. Nuyens are with the Department of Cardiovascular Sciences, University of Leuven, Leuven, Belgium. seconds), therefore, the dynamics of the heart need to be taken into account. With the standard reconstruction approach (FDK) [2] an average over the whole heart cycle is computed without providing temporal information. Consequently, a motion estimation and compensation algorithm for the heart chambers is required in order to permit temporal heart wall analysis in three dimensions.

\section{B. State-of-the-Art}

A variety of algorithms deal with the estimation of cardiac motion from a rotating C-arm system. Different approaches for recovering ventricular shapes from angiographic data using angiographic systems can be found in the literature. The reconstruction of the ventricular shape based on two orthogonal acquired 2-D projection images with a biplane C-arm system has been presented by the group of Medina. In one paper, the ventricle is formed as an ellipsoid and embedded in a Markov Random Field framework [3]. Ventricular shape reconstruction from multi-view X-ray projections has been presented by Moriyama et al. [4]. They represent time-varying 3-D shapes using uniform B-spline functions to recover the ventricular shape in 3-D. However, both methods provide only a surface model with no structural information of the ventricle, such as papillary muscles, which could be visualized in a tomographic reconstruction.

Different tomographic reconstruction algorithms attempt to improve temporal resolution. That means, several volumes corresponding to different states of the heart are reconstructed. The different states of the heart are identified by an electrocardiogram (ECG) acquired synchronous with the acquisition. A relative heart phase, commonly denoted as a percentage between two successive R-peaks, is assigned to each projection. The reconstruction is performed with the subset of the projections that lie inside a certain ECG window centered at the favored heart phase [5]. However, for the heart chambers the ECG-gated projection data of a single sweep leads to prominent streak artifacts and a poor signal-to-noise ratio. Consequently, multiple sweeps of the $\mathrm{C}$-arm have to be performed to acquire enough projections for each heart phase [6], [7]. But, the longer imaging time results in a higher contrast burden and radiation dose for the patient. In recent years, approaches dealing with undersampled projection data from ECG gating, so-called compressed sensing (CS) algo- 
rithms were published [8]. A variety of algorithms minimizes an objective function related to the total variation (TV) [9]. In one approach called prior image constrained compressed sensing (PICCS), a-priori information of the same object is incorporated into the reconstruction [10], [11]. The PICCS algorithm was recently applied to interventional images using angiographic data from a C-arm system [12], [13]. In general, volumes reconstructed with $\mathrm{TV}$ regularization have an artificial look and are highly smoothed compared to a standard FDK reconstruction. They are piecewise constant in homogenous regions and can lose small structures [14].

In this paper, we present an iterative motion estimation and compensation algorithm that uses projection data acquired during one single $\mathrm{C}$-arm sweep. It uses the ECG information to parametrize a 4-D motion field that maps the sum of all ECGgated FBP reconstructed volumes to a target heart phase by a combined multiple heart phase registration (CMHPR). This is done by correlating the combination of all ECG volumes to one reference volume reconstructed with the PICCS and the iTV algorithm.

\section{Combined Multiple Heart Phase Registration (CMHPR)}

The presented motion estimation and compensation scheme is built up from different components: First, a reference volume needs to be generated. Second, the ECG-gated volumes representing different heart phases are reconstructed with a standard FBP algorithm. The final volume is defined by the sum over the deformed ECG-gated volumes. The deformation depends on the 4-D motion vector field (MVF). The MVF is iteratively updated by minimizing an objective function.

An overview of the algorithm is given in Figure 1. A more detailed description of the individual steps is given in the following sections.

\section{A. Reference Volume Reconstruction}

First, a reference image $f_{r}(\boldsymbol{x})$ is reconstructed at a selected heart phase with a few-view reconstruction algorithm taking care of the sparse sampling condition. The reference volume needs to provide a low artifact level with minor streak artifacts, but needs to represent sharp edges of the endocardial wall. As a first attempt, the prior image constrained compressed sensing (PICCS) and the improved total variation (iTV) algorithm are used [10], [15], [16]. As prior volume for the PICCS reconstruction, a FBP reconstruction with data from a whole short-scan is used. The objective function is minimized in an alternating manner, the raw data constraint is minimized and in the second step the sparsity cost function is optimized. In order to ensure that the raw data cost function converges to the best possible value and simultaneously ensure that the sparsity constraint converges to a low value, the improved total variation (iTV) was introduced by Ritschl et al. [15], [16]. Additionally, a bilateral filter is applied to the reconstructed volume at every iteration step in order to suppress apparent noise.

\section{B. ECG-gated FBP Volume Reconstruction}

A certain number $H$ of ECG-gated volumes $f_{h}(\boldsymbol{x})$, with $h=1, \ldots, H$ at specific heart phases are reconstructed with an FDK reconstruction algorithm. The ECG-gating is performed by inserting a weighting function $\lambda$ into the standard FDK approach. Let $q_{h} \in[0,1]$ be the relative heart phase at which the reconstruction shall be carried out. The volume $f_{h}(\boldsymbol{x})$ represents one heart phase from $1, \ldots, H$. The ECG-gated FDK reconstruction $f_{h}(\boldsymbol{x}): \mathbb{R}^{3} \rightarrow \mathbb{R}$ at voxel $\boldsymbol{x} \in \mathbb{R}^{3}$ is given by

$$
f_{h}(\boldsymbol{x})=\sum_{i=1}^{N} \lambda\left(i, q_{h}\right) \cdot w(i, \boldsymbol{x}) \cdot p_{F}(i, A(i, \boldsymbol{x})),
$$

where $N$ is the number of projection images, $w: \mathbb{N} \times \mathbb{R}^{3}$ is the FDK distance weight and $p_{F}(i, \boldsymbol{u}): \mathbb{N} \times \mathbb{R}^{2} \rightarrow \mathbb{R}$ is the filtered and redundancy-weighted projection data of the $i$-th image at pixel position $\boldsymbol{u}$. The pixel position is determined by the perspective projection of voxel $\boldsymbol{x}, A: \mathbb{N} \times \mathbb{R}^{3} \rightarrow \mathbb{R}^{2},(i, \boldsymbol{x}) \rightarrow$ $A(i, \boldsymbol{x})=\boldsymbol{u}$. The perspective projection $A$ can be computed via pre-calibrated projection matrices. The weighting function $\lambda$ can be a cosine- or rectangular-window [17]. Here, we use a strict rectangular gating function of minimal width, i.e. only one view per heart cycle is considered.

\section{Final Volume Reconstruction}

The final volume is defined as a sum volume $f(\boldsymbol{x}, \boldsymbol{s})$ consisting of the deformed ECG volumes $f_{h}\left(\boldsymbol{x}+\boldsymbol{s}_{h, \boldsymbol{x}}\right)$ with motion vector $s_{h, \boldsymbol{x}}$ at heart phase $h$ and location $\boldsymbol{x}$ :

$$
f(\boldsymbol{x}, s)=\sum_{h=1}^{H} f_{h}\left(\boldsymbol{x}+\boldsymbol{s}_{h, \boldsymbol{x}}\right) .
$$

The function $f_{h}$ returns the ECG-gated, interpolated reconstructed object value at voxel $\boldsymbol{x}$ based on the motion vector $\boldsymbol{s}_{h, \boldsymbol{x}}$ for the heart phase $h$. In this paper, the motion compensated reconstruction and its derivative are based on a densely sampled motion vector field. That means for each voxel $\boldsymbol{x} \in \Omega$ at the $h$-th heart phase an individual displacement vector $\boldsymbol{s}_{h, \boldsymbol{x}} \in \mathbb{R}^{3}$ is given. $\Omega$ denotes the area where cardiac motion is assumed.

\section{Objective Function}

The 4-D motion vector field is derived by optimizing an objective function $\mathcal{L}_{N C C}(s)$ so that the negative normalized cross correlation (NCC) between the sum volume $f(\boldsymbol{x}, s)$ and the reference volume $f_{r}(\boldsymbol{x})$ is minimized. The metric ranges between $[-1,1]$. In order to define a dissimilarity measure, the negative correlation is considered here. Therefore, a value of -1 indicates a perfect positive linear relationship, a value of +1 a perfect negative linear relationship and values close to zero show no linear correlation between the volumes. The definition of the negative NCC [18], [19] combined with the 


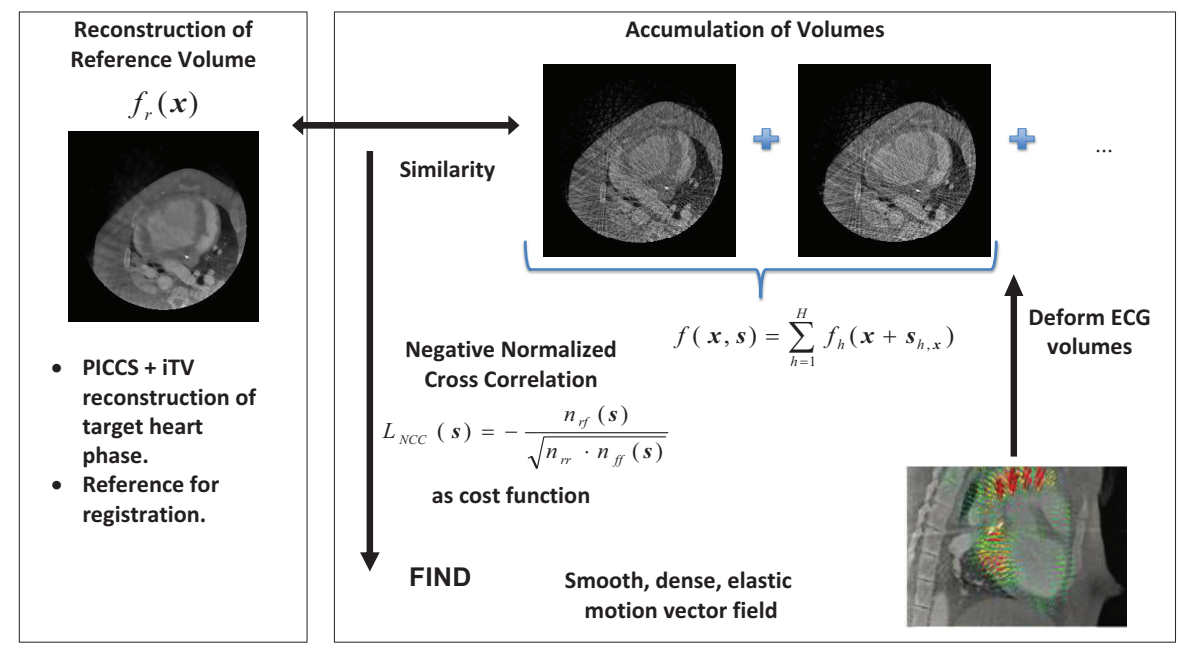

Figure 1: Schematic overview of the presented CMHPR algorithm.

computational formula for the variance [20] is given by

$$
\begin{aligned}
\mathcal{L}_{N C C}(\boldsymbol{s}) & =-\frac{n_{r f}(\boldsymbol{s})}{\sqrt{n_{r r} \cdot n_{f f}(\boldsymbol{s})}}, \text { where } \\
n_{r} & =\sum_{\boldsymbol{x} \in \Omega} f_{r}(\boldsymbol{x}) \\
n_{f}(\boldsymbol{s}) & =\sum_{\boldsymbol{x} \in \Omega} f(\boldsymbol{x}, \boldsymbol{s}) \\
n_{r r} & =\sum_{\boldsymbol{x} \in \Omega} f_{r}(\boldsymbol{x})^{2}-\frac{1}{|\Omega|} n_{r}^{2} \\
n_{f f}(\boldsymbol{s}) & =\sum_{\boldsymbol{x} \in \Omega} f(\boldsymbol{x}, \boldsymbol{s})^{2}-\frac{1}{|\Omega|} n_{f}(\boldsymbol{s})^{2} \\
n_{r f}(\boldsymbol{s}) & =\sum_{\boldsymbol{x} \in \Omega} f_{r}(\boldsymbol{x}) f(\boldsymbol{x}, \boldsymbol{s})-\frac{1}{|\Omega|} n_{r} \cdot n_{f}(\boldsymbol{s}) .
\end{aligned}
$$

\section{E. Optimization Strategy}

The objective function is minimized by a gradient based quasi-Newton method, a limited-memory Broyden-FletcherGoldfarb-Shanno optimizer (L-BFGS) [21]. Usually, the quasiNewton based methods converge in fewer iterations than gradient descent optimizers, but have a higher cost per iteration evaluation. For the optimization, the derivative of the objective function with respect to the motion vector at every heart phase and voxel is required. It can be computed as

$$
\begin{aligned}
\frac{\partial \mathcal{L}_{N C C}(\boldsymbol{s})}{\partial \boldsymbol{s}_{h, \boldsymbol{x}}}= & -\left(\frac{1}{\sqrt{n_{r r} \cdot n_{f f}(\boldsymbol{s})}} \frac{\partial n_{r f}(\boldsymbol{s})}{\partial \boldsymbol{s}_{h, \boldsymbol{x}}}-\right. \\
& \left.\frac{n_{r f}(\boldsymbol{s})}{2 \sqrt{\left(n_{r r} \cdot n_{f f}(\boldsymbol{s})\right)^{3}}} \frac{\partial n_{f f}(\boldsymbol{s})}{\partial \boldsymbol{s}_{h, \boldsymbol{x}}}\right)
\end{aligned}
$$

where the remaining components are given by

$$
\begin{aligned}
\frac{\partial n_{r f}(\boldsymbol{s})}{\partial \boldsymbol{s}_{h, \boldsymbol{x}}} & =\left(f_{r}(\boldsymbol{x})-\frac{n_{r}}{|\Omega|}\right) \frac{\partial f(\boldsymbol{x}, \boldsymbol{s})}{\partial \boldsymbol{s}_{h, \boldsymbol{x}}} \text { and } \\
\frac{\partial n_{f f}(\boldsymbol{s})}{\partial \boldsymbol{s}_{h, \boldsymbol{x}}} & =2\left(f(\boldsymbol{x}, \boldsymbol{s})-\frac{n_{f}(\boldsymbol{s})}{|\Omega|}\right) \frac{\partial f(\boldsymbol{x}, \boldsymbol{s})}{\partial \boldsymbol{s}_{h, \boldsymbol{x}}} .
\end{aligned}
$$

Finally, putting all components together we arrive at the derivative of the objective function:

$$
\begin{aligned}
\frac{\partial \mathcal{L}_{N C C}(\boldsymbol{s})}{\partial s_{h, \boldsymbol{x}}}= & -\kappa(\boldsymbol{x}, \boldsymbol{s}) \cdot \frac{\partial f(\boldsymbol{x}, \boldsymbol{s})}{\partial \boldsymbol{s}_{h, \boldsymbol{x}}} \\
= & -\kappa(\boldsymbol{x}, \boldsymbol{s}) \cdot \frac{\partial f_{h}(\boldsymbol{x}, \boldsymbol{s})}{\partial \boldsymbol{s}_{h, \boldsymbol{x}}}, \\
\text { where } \kappa(\boldsymbol{x}, \boldsymbol{s})= & \left(\frac{f_{r}(\boldsymbol{x})-\frac{n_{r}}{|\Omega|}}{\sqrt{n_{r r} \cdot n_{f f}(\boldsymbol{s})}}-\right. \\
& \left.\frac{n_{r f}(\boldsymbol{s})}{n_{f f}(\boldsymbol{s})}\left(f(\boldsymbol{x}, \boldsymbol{s})-\frac{n_{f}(\boldsymbol{s})}{|\Omega|}\right)\right) .
\end{aligned}
$$

Only $\kappa(\boldsymbol{x}, \boldsymbol{s})$ depends on the objective function and can be easily adapted if a different objective function is used. In order to guarantee a smooth motion vector field, a spatial and temporal Deriche filter is applied to the 4-D motion vector gradient. Additionally, a spatial Deriche filter is also applied to the gradient weighting term $\kappa(\boldsymbol{x}, \boldsymbol{s})$ [22], [23].

\section{F. Implementation Details}

Due to memory limitations when using a quasi-Newton optimizer and to reduce computation time, the motion vector field was first computed on downsampled volumes at a resolution of $128^{3}$ voxels and upsampled onto the final reconstructed volume size by cubic spline interpolation. The initial motion vector field was set to zero in all dimensions. The weighting parameter $\alpha$ for the PICCS algorithm was set to 0.5. The relaxation parameter $\beta$ for the iTV optimization was set to 0.8 and the iTV parameter $\omega$ to 0.8 . For the bilateral filter, 
the closeness parameter $\sigma_{d}$ was set to 0.25 and the similarity parameter $\sigma_{r}$ was set to 0.0001 . The used filter mask has a radius of 2 voxels. The Deriche filter parameter $\alpha_{D}$ for spatial filtering was heuristically set to 0.94 and for temporal filtering to 2.12 , i.e. a stronger smoothing in spatial than in temporal direction. The LBFGS optimizer of the VNL numerics library delivered with The Insight Segmentation and Registration Toolkit (ITK) ${ }^{1}$ was used for the optimization. The optimization procedure was performed until the termination criterion was reached. Here, the number of function evaluations was used which was set to 200 . The number of iterations is implicitly given by the number of function evaluations. During the linesearch of the gradient update, the objective function is evaluated more than once if we are close to a minimum, hence less iterations are performed. In order to restrict the motion vector field to a local area where the heart motion is expected, a local motion mask enforces zero motion outside the defined local area. In this first approach the mask volume defining $\Omega$ is generated manually by the user. For a later version it is planned to use an automatic heart isolation software [24], [25].

\section{EXPERIMENTAL SETUP}

The method was applied to in-vivo data sets of two porcine models $\left(p_{1}, p_{2}\right)$. Image acquisition was performed using an Artis Zee C-arm system (Siemens AG, Healthcare Sector, Forchheim, Germany). The acquisition time was $14.535 \mathrm{~s}$ capturing 381 projection images with $30 \mathrm{f} / \mathrm{s}$, and an angular increment of $0.52^{\circ}$ during one $\mathrm{C}$-arm sweep. The isotropic pixel resolution was $0.31 \mathrm{~mm} / \mathrm{pixel}(0.19 \mathrm{~mm}$ in isocenter) and the detector size $1240 \times 960$ pixel. The distance from source to detector was $1200 \mathrm{~mm}$ and from isocenter to source 750 $\mathrm{mm}$. The heart rate was synchronized with the frame-rate of the imaging acquisition through external heart pacing. The considered heart phases are equal to the number of image frames per heart cycle. For porcine model $p_{1}$ a number of 32 heart cycles and $H=12$ heart phases were imaged with an external pacing of $\approx 131 \mathrm{bpm}$. For porcine model $p_{2}$ a number of 25 heart cycles and $H=15$ heart phases were imaged with an external pacing of $\approx 105 \mathrm{bpm}$. A volume of $\sim 150 \mathrm{ml}$ contrast fluid was administered intravenously at a speed of $p_{1}=10 \mathrm{ml} / \mathrm{s}, p_{2}=6.5 \mathrm{ml} / \mathrm{s}$, with a delay of $5 \mathrm{~s}$ and 8 $\mathrm{s}$ before the X-ray rotation was started. Image reconstruction was performed on an image volume of $(25.6 \mathrm{~cm})^{3}$ distributed on a $256^{3}$ voxel grid.

\section{Results AND Discussion}

\section{A. Visual Inspection}

The reconstruction results of the 1 st porcine model $p_{1}$ at a relative heart phase of $80 \%$ are shown in Figure 2. The standard FDK reconstruction averages over all heart phases which is visible at the doubling of the catheter and the blurred endocardium edges (Fig.2a). The ECG-gated reconstruction illustrates the sharp contours of the endocardium, however

\footnotetext{
${ }^{1}$ www.itk.org
}

prominent streak artifacts are apparent (Fig.2b). Streak artifacts are reduced by the PICCS and iTV reconstruction (Fig.2c). However, the image still exhibits blurred streak artifacts and is smoothed. The presented CMHPR algorithm (Fig.2d). yields the best result. Streak artifacts are further reduced while keeping the spatial resolution. The same effects are visible in Figure 3 for a different relative heart phase of 30 $\%$ of the same porcine model $p_{1}$ and for the second porcine model $p_{2}$ for relative heart phases of $50 \%$ in Figure 4 and of $30 \%$ in Figure 5.

\section{B. Normalized Edge Response Profile}

In order to quantify the results, the normalized edge response function (similar to the edge response in Prümmer et al. [7]) of all volumes is illustrated in Figure 6. The normalized edge response profile is computed as mean edge profile of the lines indicated in Figures 2, 3, 4 and 5 and normalized to $[0,1]$. The measurements were performed for the porcine model $p_{1}$ and a relative heart phase of $80 \%$ (Fig.6a) and $30 \%$ (Fig.6b). Similar measurements were performed for the second porcine model $p_{2}$ and a relative heart phase of $50 \%$ (Fig.6c) and $30 \%$ (Fig.6d). It can be seen that the FDK reconstruction loses the edge at the endocardium border. The sharp edge is reconstructed by the ECG-gating, however, it is affected by noise and streak artifacts. The CMHPR method preserves the sharp edge similiar to the reference volume.

\section{CONClusion}

We have presented a new motion estimation and compensation algorithm by the combined multiple heart phase registration (CMHPR) which increases image quality for cardiac angiographic $\mathrm{C}$-arm data. The presented algorithm was tested on two porcine models and first results are promising. The technique reduces streak artifacts and simultaneously preserves sharp edges without getting an artificial comic-like appearance.

\section{ACKNOWLEDGMENT}

The authors gratefully acknowledge funding of the Erlangen Graduate School in Advanced Optical Technologies (SAOT) by the German Research Foundation (DFG) in the framework of the German excellence initiative.

\section{DISCLAIMER}

The concepts and information presented in this paper are based on research and are not commercially available.

\section{REFERENCES}

[1] F. Sheehan and E. Bolson, "Defining normal left ventricular wall motion from contrast ventriculograms," Physiological Measurement, vol. 24, no. 3, pp. 785-792, August 2003.

[2] L. Feldkamp, L. Davis, and J. Kress, "Practical cone-beam algorithm," Journal of the Optical Society of America A, vol. 1, no. 6, pp. 612-619, June 1984.

[3] R. Medina, M. Garreau, J. Toro, H. Breton, J. Coatrieux, and D. Jugo, "Markov random field modeling for three-dimensional reconstruction of the left ventricle in cardiac angiography," IEEE Transactions on Medical Imaging, vol. 25, no. 8, pp. 1087-1100, August 2006.

[4] M. Moriyama, Y. Sato, H. Naito, M. Hanayama, T. Ueguchi, T. Harada, F. Yoshimoto, and S. Tamura, "Reconstruction of time-varying 3-D leftventricular shape from multiview X-Ray cineangiocardiograms," IEEE Transactions on Medical Imaging, vol. 21, no. 7, pp. 773-785, July 2002. 

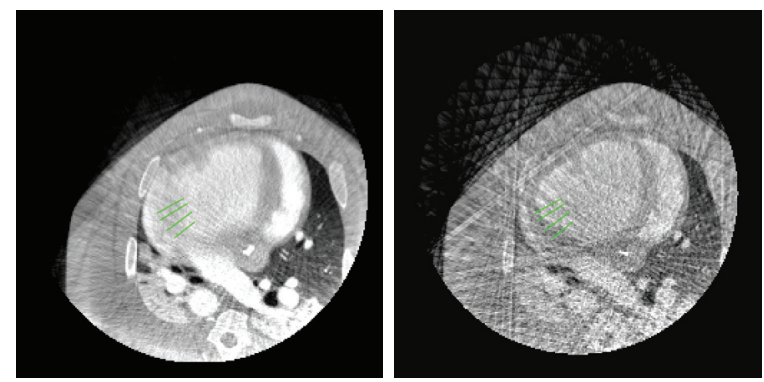

(a) Standard FDK reconstruc- (b) ECG-gated reconstruction tion.
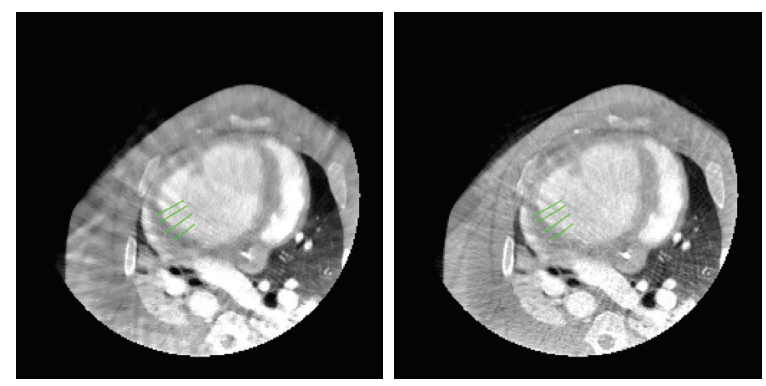

(c) PICCS+iTV reconstruction (d) Result of CMHPR algorithm. result used as reference.

Figure 2: Experimental results in porcine model $p_{1}$ of the central slice and a relative heart phase of $\approx 80 \%(\mathrm{~W} 1630$ $\mathrm{HU}, \mathrm{C} 50 \mathrm{HU}$, slice thickness $1.0 \mathrm{~mm}$ ). The ECG-gated reconstruction was windowed to be visually comparable.

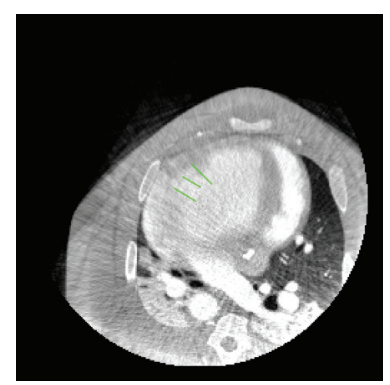

(a) Standard FDK reconstruction.

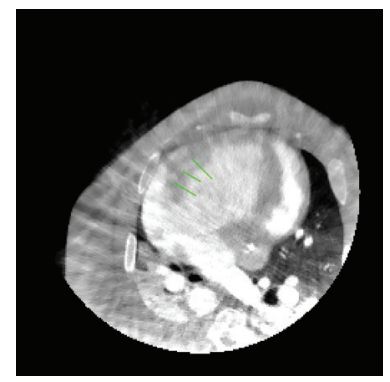

(c) Reference PICCS+iTV re- (d) Result of CMHPR algorithm. construction result.

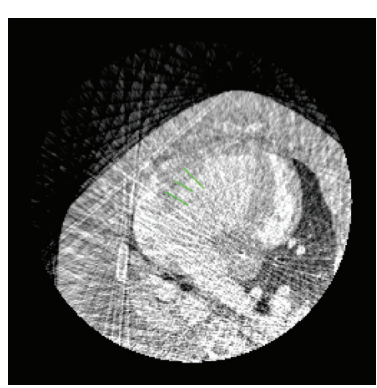

(b) ECG-gated reconstruction (32 views).

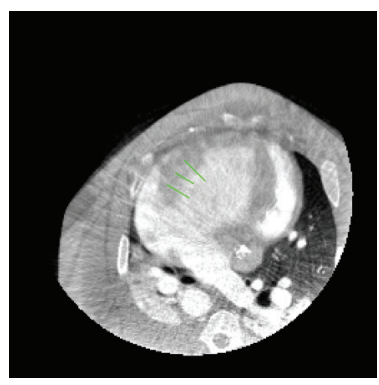

Figure 3: Experimental results in porcine model $p_{1}$ of the central slice and a relative heart phase of $\approx 30 \%(\mathrm{~W} 1630$ HU, C $50 \mathrm{HU}$, slice thickness $1.0 \mathrm{~mm}$ ). The ECG-gated reconstruction was windowed to be visually comparable.
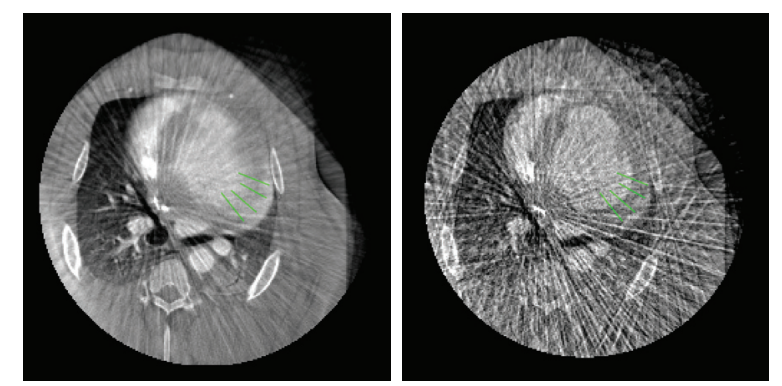

(a) Standard FDK reconstruc- (b) ECG-gated reconstruction tion.

(25 views).
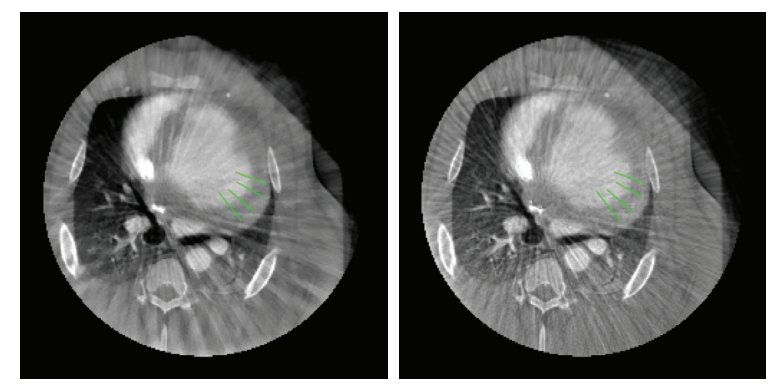

(c) PICCS+iTV reconstruction (d) Result of CMHPR algorithm. result used as reference.

Figure 4: Experimental results in porcine model $p_{2}$ of the slice 108 and a relative heart phase of $\approx 50 \%$ (W $1630 \mathrm{HU}, \mathrm{C} 50$ $\mathrm{HU}$, slice thickness $1.0 \mathrm{~mm}$ ). The ECG-gated reconstruction was windowed to be visually comparable.

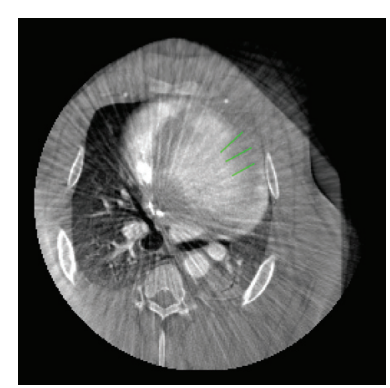

(a) FDK reconstruction.

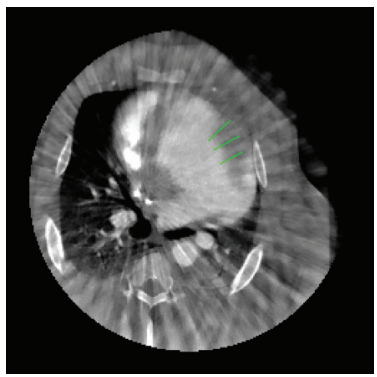

(c) PICCS+iTV reconstruction used as reference.

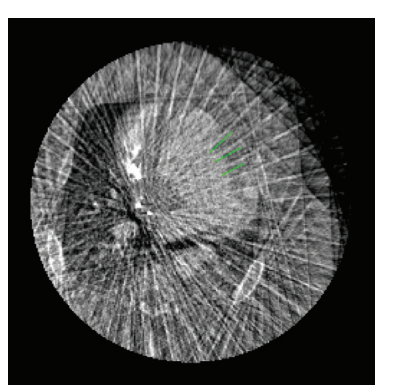

(b) ECG-gated reconstruction (25 views).

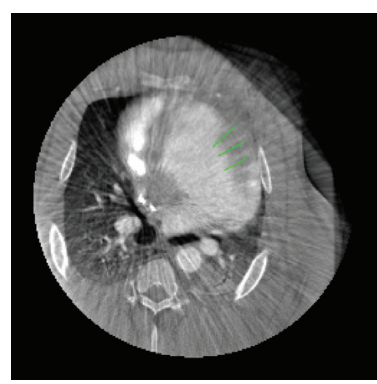

(d) CMHPR reconstruction.
Figure 5: Experimental results in porcine model $p_{2}$ of the slice 108 and a relative heart phase of $\approx 30 \%$ (W $1630 \mathrm{HU}, \mathrm{C} 50$ $\mathrm{HU}$, slice thickness $1.0 \mathrm{~mm}$ ). The ECG-gated reconstruction was windowed to be visually comparable. 


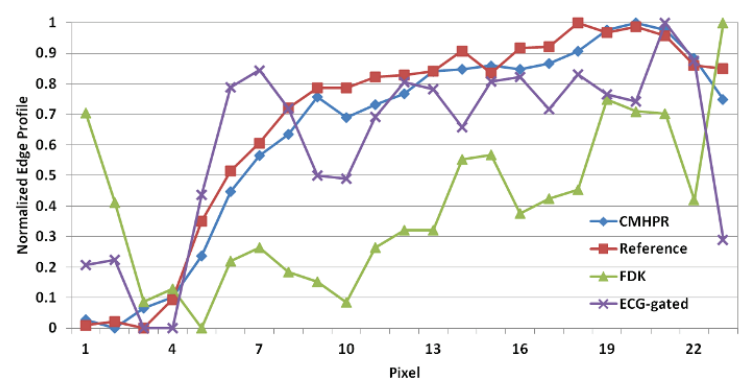

(a) Porcine model p1 and heart phase $80 \%$. Corresponding lines in Figure 2.

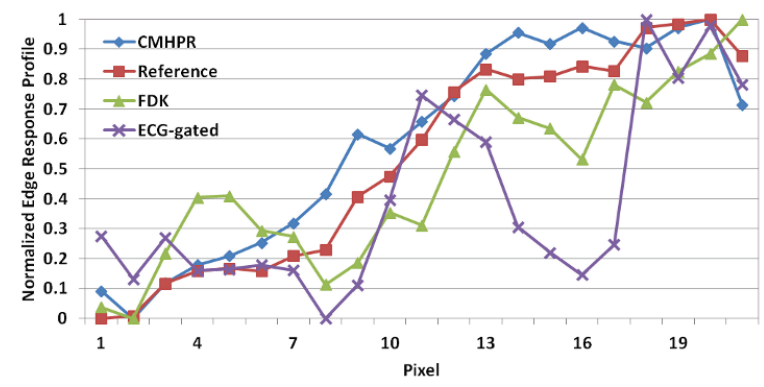

(b) Porcine model p1 and heart phase $30 \%$. Corresponding lines in Figure 3.

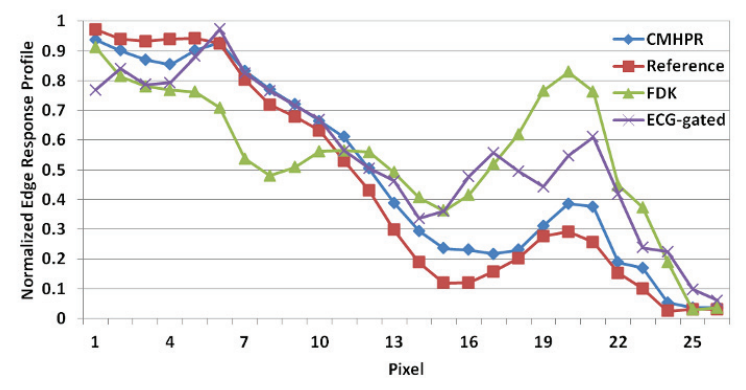

(c) Porcine model $\mathrm{p} 2$ and heart phase $50 \%$. Corresponding lines in Figure 4.

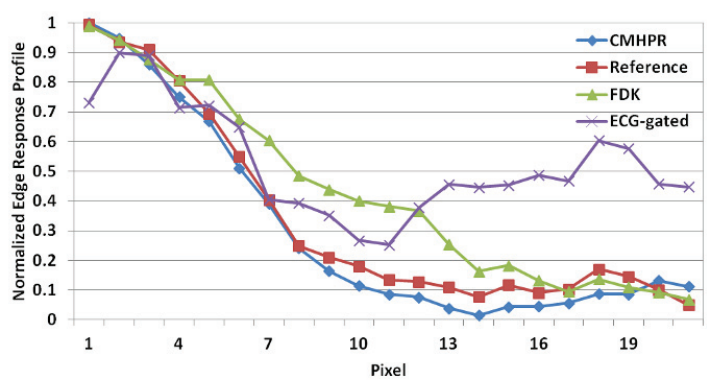

(d) Porcine model p2 and heart phase 30\%. Corresponding lines in Figure 5.

Figure 6: Normalized edge response profile of the four different reconstructions of two heart phases and the two porcine models $p_{1}$ and $p_{2}$.
[5] B. Desjardins and E. Kazerooni, "ECG-gated cardiac CT," American Journal of Roentgenology, vol. 182, no. 4, pp. 993-1010, April 2004.

[6] G. Lauritsch, J. Boese, L. Wigström, H. Kemeth, and R. Fahrig, "Towards cardiac C-arm computed tomography," IEEE Transactions on Medical Imaging, vol. 25, no. 7, pp. 922-934, July 2006.

[7] M. Prümmer, J. Hornegger, G. Lauritsch, L. Wigström, E. GirardHughes, and R. Fahrig, "Cardiac C-Arm CT: A unified framework for motion estimation and dynamic CT," IEEE Transactions on Medical Imaging, vol. 28, no. 11, pp. 1836-1849, November 2009.

[8] D. Donoho, "Compressed sensing," IEEE Transactions on Information Theory, vol. 54, no. 4, pp. 1249-1306, April 2006.

[9] E. Sidky and X. Pan, "Image reconstruction in circular cone-beam computed tomography by constrained, total-variation minimization," Physics in Medicine and Biology, vol. 53, no. 17, pp. 4777-4807, August 2008.

[10] G.-H. Chen, J. Tang, and S. Leng, "Prior image constrained compressed sensing (PICCS): A method to accurately reconstruct dynamic CT images from highly undersampled projection data sets," Medical Physics, vol. 35, no. 2, pp. 660-663, February 2008.

[11] _ _ "Prior image constrained compressed sensing(piccs)," in Proceed ings of the SPIE Medical Imaging 2008, March 2008, pp. 6856-685 618.

[12] G.-H. Chen, P. Theriault-Lauzier, J. Tang, B. Nett, S. Leng, J. Zambelli, Q. Zhihua, N. Bevins, A. Raval, and S. Reeder, "Time-resolved interventional cardiac C-arm cone-beam CT: An application of the PICCS algorithm," IEEE Transactions on Medical Imaging, vol. 31, no. 4, pp. 907-923, April 2012.

[13] P. Theriault-Lauzier, J. Tang, and G.-H. Chen, "Time-resolved cardiac interventional cone-beam CT reconstruction from fully truncated projections using the prior image constrained compressed sensing (piccs) algorithm," Physics in Medicine and Biology, vol. 57, no. 9, pp. 24612476, May 2012.

[14] Z. Tian, X. Jia, K. Yuan, T. Pan, and S. Jiang, "Low-dose CT reconstruction via edge-preserving total variation regularization," Physics in Medicine and Biology, vol. 56, no. 18, pp. 5949-5967, September 2011.

[15] L. Ritschl, F. Bergner, C. Fleischmann, and M. Kachelrieß, "Improved sparsity-constrained image reconstruction applied to clinical CT data," in IEEE Nuclear Science Symposium Conference Record (NSS/MIC), 2010, October 2010, pp. 3231-3240.

[16] — "Improved total variation-based CT image reconstruction applied to clinical data," Physics in Medicine and Biology, vol. 56, no. 6, pp. 1545-1562, Februar 2011.

[17] C. Schwemmer, C. Rohkohl, G. Lauritsch, K. Müller, and J. Hornegger, "Residual motion compensation in ECG-Gated cardiac vasculature reconstruction," in Proceedings of the second international conference on image formation in X-ray computed tomography, F. Noo, Ed., 2012, pp. 259-262.

[18] D. Russakoff, T. Rohlfing, A. Ho, D. Kim, R. Shahidi, J. Adler, and C. Maurer, "Evaluation of intensity-based 2D-3D spine image registration using clinical gold-standard data," in Biomedical Image Registration, ser. Lecture Notes in Computer Science, J. Gee, J. Maintz, and M. Vannier, Eds. Springer Berlin / Heidelberg, 2003, vol. 2717, pp. $151-160$.

[19] G. Penney, J. Weese, J. Little, P. Desmedt, D. Hill, and D. Hawkes, "A comparison of similarity measures for use in 2-D-3-D medical image registration," IEEE Transactions on Medical Imaging, vol. 17, no. 4, pp. 586-595, August 1998.

[20] D. Knuth, Seminumerical Algorithms, ser. The Art of Computer Programming. Addison-Wesley Longman, 1998, vol. 2.

[21] R. Fletcher, "A new approach to variable metric algorithms," The Computer Journal, vol. 13, no. 3, pp. 317-322, August 1970.

[22] R. Deriche, "Using canny's criteria to derive a recursively implemented optimal edge detector," International Journal of Computer Vision, vol. 1, no. 2, pp. 167-187, June 1987.

[23] — " "Fast algorithms for low-level vision," IEEE Transactions on Pattern Analysis and Machine Intelligence, vol. 12, no. 1, pp. 78-87, January 1990.

[24] Y. Zheng, F. Vega-Higuera, S. Zhou, and D. Comaniciu, "Fast and automatic heart isolation in 3D CT volumes: optimal shape initialization," in Proceedings of the First international conference on Machine Learning in Medical Imaging (MLMI), September 2010, pp. 84-91.

[25] G. Funka-Lea, C. Florin, M.-P. Jolly, R. Moreau-Gobard, R. Ramaraj, and D. Rinck, "Automatic heart isolation for CT coronary visualization using graph-cuts," in Proceedings of the IEEE International Symposium on Biomedical Imaging (ISBI) 2009, April 2006, pp. 614-617. 